\title{
Infant delivery and maternal stress during the COVID-19 pandemic: a comparison of the well-baby versus neonatal intensive care environments
}

\author{
Alona Bin-Nun $\mathbb{1}^{1,2} \cdot$ Shoshana Palmor-Haspal ${ }^{1,3} \cdot$ Francis B. Mimouni ${ }^{1,4} \cdot$ Yair Kasirer $\mathbb{1}^{1,2} \cdot$ \\ Cathy Hammerman $\mathbb{1 0}^{1,2} \cdot$ Rivka Tuval-Moshiach ${ }^{5}$
}

Received: 24 September 2020 / Revised: 10 January 2021 / Accepted: 17 February 2021 / Published online: 13 May 2021

(c) The Author(s), under exclusive licence to Springer Nature America, Inc. 2021

\begin{abstract}
Objective To describe impact of COVID-19 pandemic on stress and mood of new mothers, in particular in neonatal intensive care unit (NICU); a secondary objective was to assess whether customary social gender distancing practiced by ultra-religious Jews and Muslims offers built-in anti-stress protection.

Methods Cross-sectional, observational survey of mothers of 52 normal newborn nursery (NNB) and 52 NICU infants. In all, 86 filled all the 6 questionnaires (Demographics, COVID-19 virus experience, Mental Health Inventory, Neonatal Satisfaction Survey, Parental Stressor Scale, and Questionnaire of Coping Strategies).

Results Most mothers stated that COVID-19 pandemic had hurt social and family relationships, maternal role, and expressed stress and loneliness. Mothers of NICU infants had higher degree of helplessness. Religious social distancing was not protective. Background tendency to coping poorly with stress and depression most highly predicted stress.

Conclusion COVID-19 pandemic harms psychosocial well-being of most mothers. Detection of high-risk individuals is necessary to provide appropriate support.
\end{abstract}

\section{Introduction}

The Center for Disease Control (CDC) has recognized that, since its inception, "the coronavirus disease 2019 (COVID19) pandemic may be stressful for people" [1]. Among major potential sources of stress, the CDC singled out fear and anxiety concerning a new and little understood disease, fear about one's own health and health of loved ones, worry about isolation should one actually become ill, deterioration of one's financial situation secondary to job loss, and/or loss of support services. Social distancing has been central to the

Yair Kasirer

yairkasir@szmc.org.il

1 Shaare Zedek Medical Center, Jerusalem, Israel

2 The Faculty of Medicine of the Hebrew University, Jerusalem, Israel

3 Herzog Academic College, Jerusalem, Israel

4 Sackler Faculty of Medicine, Tel Aviv University, Tel Aviv, Israel

5 Department of Psychology, Bar Ilan University, Ramat Gan, Israel
CDC recommendations in order to contain the COVID-19 pandemic. Social distancing, which necessitates a separation of "at least 6 feet from others who are not from your household" [2], is likely to exacerbate existing feelings of isolation and loneliness.

It has also long been recognized that the delivery of a new baby may be a stressful situation. The British National Health Services (NHS) has issued a guide to new parents about how to cope with relationships after having a baby [3]. Indeed, becoming a parent is often stressful on the spousal relationship, regardless of what the relationship was before [3]. Recognized risk factors for such stress include fatigue, relative lack of sleep, significant reduction in time invested in the spousal relationship, difficulties in keeping up with friends, social isolation, hormonal surges and troughs, etc....[3]. The postnatal period is a time of life when human closeness, intimacy, and understanding are especially important.

Should the neonate be delivered prematurely or be ill, these stresses are likely to be compounded. A recent study [4] showed that both mother and father may have increased stress levels and negative feelings after premature births, which could have a negative impact on both the 
inter-spousal relationship and the parent-child interactions. Stress and negative feelings correlate with parental youth and with the severity of the baby's medical condition.

We therefore conducted the following prospective, crosssectional, observational study to describe the impact of the COVID-19 pandemic on self-described measures of stress and mood of mothers delivering infants in our institution in two separate settings: (1) that of the well-baby nursery and (2) that of the neonatal intensive care unit (NICU). Our secondary objectives were to: (1) assess whether or not delivery of a sick, preterm neonate is an additional independent stressor in the COVID-19 environment; and (2) assess whether customary social gender distancing and/or segregation practiced regularly by ultraorthodox Jewish [5] and devout Muslim mothers [6] offers a built-in protective anti-stress defensive mechanism during COVID-19 times.

\section{Methods}

The study was approved by our local Institutional Review Board and all mothers signed a written consent form to indicate their agreement to participate in the study. It is a prospective, observational, non-interventional survey, in which we intended to recruit the mothers of approximately 50 infants admitted to one of the normal newborn nurseries (NNB) and 50 infants admitted to the NICU in our institution between 1 April 2020 and 30 May 2020. Our NICU is a modern level III NICU, which admits approximately 1000 sick neonates per year, and serves a general population of nearly 18,000 deliveries per year. There are a limited number of 1 mother/infant dyad in full rooming in, while most mothers are housed in 2-3 patients' rooms. Since the COVID-19 pandemic started, many hospital policies were modified or adjusted. Babies were allowed to breastfeed at the breast, and were not separated from a mother with COVID-19. However, from this study we excluded all mothers with active COVID-19 disease at the time of delivery. All mothers had a COVID-19 exposure medical history taken and had their temperature assessed upon admission. There were instructed to wear mask at all time during the hospital admission except for brief periods of eating or washing, and were instructed to wash their hands prior to any kind of baby handling. No more than one visitor at a time was allowed, and only belonging to the same nuclear family (spouse or child). In the NICU, distancing of at least $2 \mathrm{~m}$ between babies was implemented at all times, but was not possible in the well-baby nurseries, thus rooming in was encouraged at all times, even in multiple patients' (up to 4, but mostly 2 ) rooms.

Potential consecutive candidates for inclusion were approached. If they consented to participate, they were invited to fill out anonymously a standard set of 6 questionnaires, the Hebrew version of which was translated and validated by Florian and Drori [7]. The abbreviated version of the questionnaire was developed in Israel by Isaac [8]. Questionnaires were filled on the patients' own smartphones using the Qualtrics XM software (Qualtrics, Provo, Utah). In the event that the mother did not possess a smartphone, she received a series of 6 printed questionnaires to fill in and return by mail. In case of Arabicspeaking mothers, the questionnaire was translated by a certified translator. The 6 questionnaires involved are:

1. A demographics questionnaire. This questionnaire included details regarding the mother's education (years of formal education) and financial situation (self-described, and reported in a scale from 1 (poor) to 5 (rich)), number of children in the home, her marital status, and self-defined level of religiosity. Particular emphasis was made about whether or not the mother practiced "religious distancing" (no touch with any other man who is not her husband) on a daily basis (self-defining themselves as Ultra-Orthodox for Jews, and devout for Muslims) or not (Orthodox, i.e., religious, but tolerating touch among sexes, traditional (an equivalent of Conservatist Judaism in the USA), or secular, the latter valid for non-religious Muslims as well) $[5,6]$.

2. A COVID-19-related questionnaire. This questionnaire included 17 items compiled for the purpose of this study in order to examine the parent's perception of the consequences of the COVID-19 for herself, for her family members, and for her perceived attitude of the staff.

3. A Mental Health Inventory (MHI) questionnaire. This questionnaire describes subjective feelings of the individual, along with a description of mental and somatic symptoms which are correlated with depression, anxiety and well-being experienced by the mother during her stay in the hospital. The MHI questionnaire includes 15 items -9 of which examine mental distress, 6 which examine mental well-being. $(1=$ at no time, $6=$ all the time.) The questionnaire total score is the average of the answers on each subscale [8].

4. A Neonatal Satisfaction Survey (NSS) questionnaire [9]. This questionnaire includes parents' reference to their degree of satisfaction with the care given to them in preterm labor. The NSS-8 questionnaire includes 51 items and two general satisfaction assessment questions. The questionnaire consists of eight factors relating to care and concern, physicians, visits, the physical environment and a variety of preterm infants, parental anxiety information, and discharge from the ward. The questionnaire was found to be well 
validated [9]. For the purpose of the present study, items from factors irrelevant to the present study (related to siblings and physical conditions in the ward) were removed to facilitate the parents completing the questionnaire. Each item is rated on a Likert scale between 1 and 5 , with a higher score indicating a higher level of satisfaction.

5. A Parental Stressor Scale questionnaire: Neonatal Intensive Care Unit (PSS-NICU) [10] (Miles et al., 1993). This questionnaire includes 46 items, which relate to four areas, in addition to another item that relates to general stress. The four areas are: sights and sounds, baby looks, parent-baby relationships, and staff. The parent is asked to fill in with respect to each item how stressful it is for him from $1=$ not stressful at all to $5=$ most stressful. If a parent does not experience one of the items in the questionnaire, she marks "irrelevant." The questionnaire is widely used in preterm infants and has been found to have good structural validity, and an internal consistency of 0.89 . Reliability, in the form of internal consistency, has been shown to be acceptable $(>0.70)$ for the PSS: NICU [11, 12]. Cronbach's alpha coefficients ranged from 0.73 to 0.81 for the sights and sounds subscale, 0.83 to 0.90 for the infant appearance subscale, 0.83 to 0.90 for the parent-infant relationship subscale, and 0.89 to 0.94 for the total scale.

6. Questionnaire of Coping Strategies [11]. The questionnaire examines coping strategies in stressful situations. We used an abridged version (30 items) of this questionnaire. Each item is rated on a Likert scale between 1 and 5 , with a higher score indicating better handling. High reliability and validity have been found in the use of this tool in Hebrew $[12,13]$.

\section{Statistical analyses}

For this pilot study, sample size determination was based on a convenience sample of approximately 50 mothers in each group. The Minitab Statistical Package, version 16 (Minitab, State College, PA) was used for analyses. Descriptive statistics were calculated for demographic data and all psychosocial variables studied. Data were tested for normality and continuous variables were expressed as mean \pm standard deviation, or median and range as suggested by the presence or absence of normality. Categorical variables were expressed as frequencies and percent. Association between two categorical variables was tested using Chisquare test or Fisher's exact test, while the comparison of continuous variables between groups was performed using either $t$-test or non-parametric Kruskal-Wallis test. The variables that were found to be significantly associated with a dependent variable in univariable analyses were then entered into a multivariate model of multiple regression. All tests were two-tailed, and a $P$-value of less than 0.05 was considered statistically significant.

\section{Results}

During the study period, the mothers of 52 consecutive infants in the NICU and 52 consecutive infants in the NNB were approached and agreed to participate into the study, but only 86 actually filled and returned the questionnaires.

Table 1 describes selected demographic variables collected in the population. In brief, mothers had an average age of 31 years, most of them multiparous, a majority Jewish, and mostly orthodox or ultraorthodox. Most mothers were highly educated, and defined themselves as slightly above average socioeconomic status. Very few [2] had contracted COVID-19 in the recent past, although 5 had been exposed to the virus. Approximately $8 \%$ had personally experienced quarantine.

Table 2 (second column) describes the background psychological condition/affect in both groups of mothers (prior to becoming pregnant). Mothers described themselves as higher than average stable, were in general happy, and about average in terms of having trouble coping with stressful situations or feeling usually depressed or under stress. When stressed, very few claimed using alcohol or medications, using jokes, or expressing emotional distress. They asserted seeking emotional support frequently, developing a plan of action, and more often than not prayed when stressed.

Table 3 (second column) depicts the self-described psychosocial impact of COVID-19 pandemic on mothers from both sites of admission. A majority of mothers stated that the COVID-19 pandemic had adversely affected their social relationships, their family relationships, their perceived maternal role in the family, and most mothers expressed feeling anxiety for their family's health, and felt lonely. A third of the mothers described that they had experienced a decrease in spousal support as a consequence of the pandemic.

Table 4 (second column) describes changes in affect since the beginning of the COVID-19 pandemic. Mothers scored themselves above average in terms of feeling calm and relieved, relaxed, confident, or on the contrary nervous, stressed, sad, anxious, worried and restless; mothers of NICU infants expressed more worry to contaminate their baby and a higher degree of helplessness.

Table 5 (second column) summarizes the impact of the delivery (supposedly regardless of the pandemic) on familial-social relationships. While approximately half of the mothers indicated that the delivery of the infant had hurt 
Table 1 Selected demographic data.

\begin{tabular}{|c|c|c|c|c|}
\hline & Whole group & NICU & NNB & $\begin{array}{l}P \text {-value } \\
(\mathrm{NICU} \text { vs NNB) }\end{array}$ \\
\hline Maternal age (years) & $30.0 \pm 5.8$ & $28.6 \pm 6.6$ & $31.3 \pm 4.6$ & 0.03 \\
\hline Parity & $3.1 \pm 2.4$ & $2.8 \pm 2.8$ & $3.3 \pm 2.1$ & 0.415 \\
\hline Gravidity & $3.6 \pm 3.0$ & $3.6 \pm 3.6$ & $3.6 \pm 2.2$ & 0.988 \\
\hline Birth weight (g) & $2689 \pm 913$ & $2116 \pm 822$ & $3326 \pm 484$ & 0.001 \\
\hline $\begin{array}{l}\text { Cesarean/vaginal deliveries } \\
\text { ratio }(\%)\end{array}$ & $21.20 \%$ & $11 / 41(26.8 \%)$ & $7 / 44(15.9 \%)$ & 0.218 \\
\hline Assisted reproductive technology & $14.10 \%$ & $7 / 40$ & $5 / 40$ & 0.389 \\
\hline Length of stay (days, median) & $18.0 \pm 32.5$ & 14 & 2.75 & 0.001 \\
\hline Education (years) & $14.9 \pm 2.7$ & $14.8 \pm 2.9$ & $14.8 \pm 2.6$ & 0.919 \\
\hline Religiosity $(n)$ & & & & 0.486 \\
\hline Secular & 1 & 4 & 6 & \\
\hline Traditional & 9 & 4 & 5 & \\
\hline Religious & 38 & 10 & 28 & \\
\hline Ultra-religious & 29 & 23 & 6 & \\
\hline Religion $(n)$ & & & & 0.613 \\
\hline Jewish & 83 & 40 & 43 & \\
\hline Muslim & 3 & 1 & 2 & \\
\hline Christian & 0 & 0 & 0 & \\
\hline Other & 1 & 0 & 0 & \\
\hline $\begin{array}{l}\text { Used to distancing on religious } \\
\text { grounds }\end{array}$ & $59 / 86(68.6 \%)$ & $32 / 41(78 \%)$ & $27 / 45(60 \%)$ & 0.069 \\
\hline Economic status & $3.1 \pm 0.7$ & $3.2 \pm 0.8$ & $3.1 \pm 0.6$ & 0.37 \\
\hline Self-reported health index & $4.6 \pm 0.6$ & $4.8 \pm 0.5$ & $4.4 \pm 0.6$ & 0.005 \\
\hline $\begin{array}{l}\text { Known COVID-19 exposure, no } \\
\text { infection }\end{array}$ & $5 / 86(5.8 \%)$ & $5 / 42(11.9 \%)$ & $0 / 42(0.0 \%)$ & 0.05 \\
\hline $\begin{array}{l}\text { Known COVID-19 exposure and } \\
\text { infection }\end{array}$ & $2 / 86(2.3 \%)$ & $0 / 42(0 \%)$ & $2 / 44(4.6 \%)$ & 0.05 \\
\hline Quarantine experience & $7 / 86(8.1 \%)$ & $5 / 42(11.9 \%)$ & $2 / 44(4.6 \%)$ & 0.212 \\
\hline
\end{tabular}

social relationships, only about one-third described damage to family relationships and a majority described that their role in the family had been hurt by the delivery of the new baby.

We then compared (Tables 1-5, columns 3 and 4) NICU mothers to NNB mothers. In brief, mothers from the NICU were younger, and self-reported a better general health condition. They did not significantly differ from mothers of the NNB in other demographic parameters or in background psychological condition/affect in both groups of mothers (prior to becoming pregnant) except that they stated less often that they would use joke when stressed or express distress under stress. Infant birth weight was smaller and length of hospital stay was longer in NICU infants than in NNB infants. Mothers from the NICU had been more often SARS-Cov-2 exposed or infected, but there were no significant differences in quarantine experience between the two groups. There were no significant differences between the two groups in self-described changes in affect since the beginning of the COVID-19 pandemic, except that mothers of NICU infants expressed more worry to contaminate their baby and experienced a higher degree of helplessness.

In stepwise backward regression analysis, while taking into account the stress measures as the dependent variable (the Mental Health Inventory score) and maternal age, gravidity, religion and religiousness, customary practice of religious distancing, educational status, socioeconomic status, previous experience of quarantine, background difficulties coping with stress, background tendency to depression, NICU vs NNB admission, only background tendency to coping poorly with stress $(P=0.001)$, and background tendency to depression $(P=0.004)$ remained in the final analysis, while young maternal age reached a $P$ value of 0.07 .

\section{Discussion}

In this study, we confirmed our hypothesis that, following the delivery of an infant, a majority of mothers stated that 
Table 2 Psychological condition/affect pre-pregnancy maternal background (each item is rated on a Likert scale between 1 and 5, a higher score indicating a higher level of consistency of the answer with the question).
Table 3 Self-described social/ familial impact of COVID-19 pandemic by site of admission.

\begin{tabular}{lccll}
\hline & $\begin{array}{l}\text { Whole study } \\
\text { population }\end{array}$ & NICU & NNB & $\begin{array}{l}P \text {-value (NICU } \\
\text { vs NNB) }\end{array}$ \\
\hline Is emotionally stable & $3.8 \pm 1.4$ & $3.9 \pm 1.4$ & $3.7 \pm 1.4$ & 0.588 \\
Has troubles coping with stress & $2.5 \pm 1.2$ & $2.6 \pm 1.4$ & $2.5 \pm 1.0$ & 0.721 \\
Is happy in general & $3.9 \pm 1.3$ & $3.8 \pm 1.3$ & $4.0 \pm 1.2$ & 0.434 \\
Is depressed under stress & $2.5 \pm 1.3$ & $2.6 \pm 1.3$ & $2.3 \pm 1.4$ & 0.453 \\
Uses alcohol or medications when & $1.13 \pm 0.5$ & $1.1 \pm 0.4$ & $1.1 \pm 0.5$ & 0.718 \\
stressed & $1.9 \pm 0.9$ & $1.7 \pm 0.8$ & $2.1 \pm 1.0$ & 0.039 \\
Jokes when stressed & $1.9 \pm 1.0$ & $1.7 \pm 0.8$ & $2.1 \pm 1.0$ & 0.039 \\
Expresses emotional distress when & & & & \\
stressed & $3.1 \pm 0.9$ & $3.3 \pm 0.9$ & $3.0 \pm 0.8$ & 0.252 \\
Is fatalist when stressed & $3.2 \pm 0.9$ & $3.2 \pm 0.9$ & $3.1 \pm 0.9$ & 0.730 \\
Seeks for emotional support when & & & & \\
stressed & $2.9 \pm 0.9$ & $2.9 \pm 0.9$ & $2.9 \pm 1.0$ & 0.928 \\
Plans action when stressed & $2.7 \pm 1.1$ & $2.9 \pm 1.1$ & $2.5 \pm 1.1$ & 0.099 \\
Prays when stressed & & &
\end{tabular}

\begin{tabular}{lllll}
\hline Damage to & Whole group & NICU & NNB & $P$-Value (NICU vs NNB) \\
\hline Social relationships & $64 / 85(75 \%)$ & $32 / 42(76.2 \%)$ & $32 / 43(74 \%)$ & 0.982 \\
Family relationships & $58 / 85(68 \%)$ & $26 / 42(62 \%)$ & $32 / 43(74 \%)$ & 0.249 \\
Maternal role & $56 / 84(67 \%)$ & $25 / 42(59.5 \%)$ & $31 / 42(73.8 \%)$ & 0.247 \\
Family health anxiety & $81 / 86(94 \%)$ & $39 / 42(92.9 \%)$ & $42 / 44(95.4 \%)$ & 0.854 \\
Loneliness & $64 / 86(74 \%)$ & $31 / 42(73.8 \%)$ & $33 / 44(75 \%)$ & 0.980 \\
Social support & $67 / 86(78 \%)$ & $31 / 42(73.8 \%)$ & $36 / 44(81.8 \%)$ & 0.198 \\
Spousal support & $28 / 85(33 \%)$ & $17 / 42(40.4 \%)$ & $11 / 43(25.6 \%)$ & 0.632 \\
\hline
\end{tabular}

Table 4 Changes in affect since the beginning of the COVID-19 pandemic (each item is rated on a Likert scale between 1 and 5 , a higher score indicating a higher level of consistency with the question).

\begin{tabular}{lllll}
\hline Feeling & Whole group & NICU & NNB & $P$-value \\
\hline Calm and relieved & $3.3 \pm 1.8$ & $3.4 \pm 1.3$ & $3.1 \pm 1.1$ & 0.199 \\
Relaxed & $3.2 \pm 1.1$ & $3.4 \pm 1.1$ & $3.1 \pm 1.0$ & 0.278 \\
Confident & $3.0 \pm 1.2$ & $3.0 \pm 1.2$ & $3.0 \pm 1.2$ & 0.782 \\
Nervous & $2.7 \pm 1.0$ & $2.5 \pm 0.9$ & $2.8 \pm 1.1$ & 0.107 \\
Stressed & $3.1 \pm 1.1$ & $2.9 \pm 1.2$ & $3.3 \pm 1.0$ & 0.164 \\
Anxious & $3.1 \pm 1.4$ & $3.3 \pm 1.4$ & $2.8 \pm 1.4$ & 0.114 \\
Worried & $3.5 \pm 1.09$ & $3.6 \pm 1.1$ & $3.5 \pm 1.1$ & 0.766 \\
Restlessness & $3.0 \pm 1.3$ & $3.1 \pm 1.3$ & $2.8 \pm 1.2$ & 0.224 \\
Sad & $2.6 \pm 1.1$ & $2.6 \pm 1.1$ & $2.6 \pm 1.2$ & 0.727 \\
Helplessness & $3.2 \pm 1.7$ & $3.8 \pm 1.4$ & $2.5 \pm 1.8$ & 0.001 \\
Worried about & $3.6 \pm 1.7$ & $3.1 \pm 1.8$ & $4.0 \pm 1.6$ & 0.027 \\
infecting baby & & & & \\
\hline
\end{tabular}

the COVID-19 pandemic had adversely affected their social relationships, their family relationships, their perceived maternal role in the family, and most mothers expressed feeling anxiety for their family's health, as well as
Table 5 Effect of delivery on psychosocial variables by site of admission.

\begin{tabular}{lllll}
\hline Damage to & Whole group & NICU & NNB & $P$-value \\
\hline $\begin{array}{l}\text { Social } \\
\text { relationships }\end{array}$ & $45 / 84(54 \%)$ & $21 / 42(50 \%)$ & $24 / 42(57 \%)$ & 0.777 \\
$\begin{array}{l}\text { Family } \\
\text { relationships }\end{array}$ & $30 / 85(37 \%)$ & $14 / 42(33.3 \%)$ & $16 / 43(37.2 \%)$ & 0.928 \\
$\begin{array}{l}\text { Maternal role } \\
57 / 84(68 \%)\end{array}$ & $26 / 42(61.9 \%)$ & $31 / 42(73.8 \%)$ & 0.350 \\
\hline
\end{tabular}

loneliness. In addition, a third of mothers in both groups felt a decrease in spousal support as a consequence of the pandemic. Obviously, we wish we had a control group prior to the COVID-19 pandemic, which would have allowed us to truly compare scores before and during pandemics. Nevertheless, our findings were true whether or not healthy (NNB) or less healthy (NICU) babies had been delivered, with the only difference between both groups of mothers being that mothers of NICU infants expressed more worry about infecting their baby and a greater degree of helplessness. They also used less joking as a strategy of coping, an indicator of greater stress. We speculate that mothers of healthy babies, who are encouraged to spend all their time 
with their baby (rooming in is available in our institution upon request), feel more empowered to take exclusive care of their baby, and are less likely to experience feelings of helplessness than mothers of preterm infants taken care of essentially by nurses and physicians. It is also possible that during their short stay in the hospital mothers of healthy babies are less likely to contract COVID-19 as opposed to most discharged NICU moms who travel back and forth from their home to the hospital frequently, are more exposed to people outside the hospital, and for that reason may be more afraid of contracting the disease. The extraordinary routine measures to prevent nosocomial infections in NICUs coupled with the knowledge of the sicker infants increased susceptibility probably highlight the fear of disease transmission and probably explain why NICU mothers expressed more worry about contaminating their baby. Nevertheless, Hagen et al. [9] have shown that parents who experience a premature birth and/or trauma in childbirth that requires hospitalization in the NICU may feel existential anxiety about their child's fate, depression, and uncertainty about its future development. In addition they may feel guilty for not being able to complete the months of pregnancy and/or for not being able to prevent pain and suffering from their child [14], although this may be alleviated if they are allowed to care for their baby due to its complex medical condition $[15,16]$. Although preterm infants can survive without the involvement of their parents, their optimal physical, cognitive, and emotional development is found to occur during their positive and loving interaction with their parents. Since the COVID-19 pandemic started, we have been using a policy of one single parent visiting at a time, and it is possible that this policy may also have impacted on spousal relationships.

We also confirmed that regardless of the COVID-19 pandemic, mothers indicated that the delivery of a new baby alone was stressful and impacted negatively on social relationships, as described previously [17]. Supposedly, these specific answers were provided "regardless of the COVID-19 pandemic", but since we do not have a historical control group prior to the pandemic, it can only be assumed that mothers were able to distinguish between the stress caused by the delivery and that caused by the pandemics.

Contrary to our hypothesis, the customary practice of ultraorthodox Jewish custom of prohibiting non-spousal touch, also practiced by devout Muslim women, did not offer a stress-protecting defense. We speculate that this apparent lack of protective effect is due to the fact that religious prohibition of touch in Judaism and Islam is in essence different from social distancing promoted during the COVID-19 pandemic. Both Ultraorthodox Jewish and devout Muslim men are prohibited from touching women who are not their wives, but there is no specified distance limitation between men and women as long as they do not touch each other. Furthermore, there is no religious prohibition of touch between men or between women. During the COVID-19 pandemic it has been nearly universally recommended that people not belonging to the same nuclear family stay at a 6 feet (approximately $2 \mathrm{~m}$ ) distance from each other, regardless of their gender [6]. Thus the COVID19 social restrictions may in the end have been perceived by both secular and religious women in a similar manner. We cannot however assume that COVID-19-related stress was only due to distancing, rather than to social isolation and risk of disease, morbidity and mortality, and changes in hospital policy (masks, number of visitors, fever screening, etc....), which might have affected stress level in multiple ways.

From our multivariable regression analysis, it was obvious that previous mental state of the mothers and their ways of coping were maybe the most important predictors of stress. This has been previously described in a series of recent studies that showed that parental well-being affects the well-being of their children [18].

We conclude that the COVID-19 pandemic has caused and probably continues to cause great harm to the psychosocial well-being of new mothers, and consequently of their families. We thus suggest that both well-baby nursery and NICU caretakers be at high alert for detecting at-risk individuals and their families in order to provide them with empathy, reassurance, and if necessary psychosocial support as needed.

Author contributions $\mathrm{AB}-\mathrm{N}$ contributed to the concept and its realization, statistical analyses and writing of the manuscript. SP-H contributed to the data collection and organization, and to the writing of the manuscript. FBM contributed to the concept and its realization, statistical analyses and writing of the manuscript. YK and $\mathrm{CH}$ contributed to the concept and critical review of the manuscript. RT-M contributed to statistical analyses and critical review of the manuscript. All authors reviewed the manuscript for important intellectual contents and approved the final version. All authors agree to be accountable for all aspects of the work.

Funding The study has no funding and no financial support from any company or any grant.

\section{Compliance with ethical standards}

Conflict of interest The authors declare no competing interests.

Publisher's note Springer Nature remains neutral with regard to jurisdictional claims in published maps and institutional affiliations.

\section{References}

1. The Center for Disease Control. Coping with stress. https://www. cdc.gov/coronavirus/2019-ncov/daily-life-coping/managingstress-anxiety.html (2020). Accessed 26 Aug 2020. 
2. Centers for Disease Control and Prevention. Social distancing. https://www.cdc.gov/coronavirus/2019-ncov/prevent-getting-sick/ social-distancing.html (2020). Accessed 26 Aug 2020.

3. The National Health Services. Relationships after having a baby. https://www.nhs.uk/conditions/pregnancy-and-baby/rela tionships-after-a-baby/ (2020). Accessed 26 Aug 2020.

4. Ionio C, Mascheroni E, Colombo C, Castoldi F, Lista G. Stress and feelings in mothers and fathers in NICU: identifying risk factors early interventions.Prim Health Care Res Dev. 2019;20:e81 1-7.

5. Wikipedia. Negiah. https://en.wikipedia.org/wiki/Negiah (2020). Accessed 31 Aug 2020.

6. Wikipedia. Islam and gender segregation. https://en.wikipedia. org/wiki/Islam_and_gender_segregation\#: :text $=$ There $\% 20$ have $\% 20$ been\%20fatwas\%20which,is\%20Muslim\%20or\%20non\% 2DMuslim (2020). Accessed 31 Aug 2020.

7. Florian V, Drori Y. Mental Health Index (MHI): psychometric features and normative data among the Israeli population. Psychology. 1990;2:26-35.

8. Izsak R. How young people adapt to the army framework: the officer's functioning as a solid base and his contribution to the mental wellbeing and military functioning of the soldier. Unpublished Doctoral Dissertation. Ramat Gan: Bar Ilan University; 2002 (in Hebrew).

9. Hagen I.H, Iversen V.C, Nessert E, Orner R, Svindseth M.F. Parental satisfaction with neonatal intensive care units: a quantitative cross-sectional study.BMC Health Serv Res. 2019;19:37
10. Miles MS, Funk SG, Carlson J. Parental Stressor Scale: neonatal intensive care unit. Nurs Res. 1993;42:148-52.

11. Carver CS, Scheier MF, Weintraub JK. Assessing coping strategies: a theoretically based approach. J Personal Soc Psychol. 1989;56:267-83.

12. Gilbar O, Ben-Zur H. Cancer and the family caregiver: distress and coping. Springfield, IL: Charles C. Thomas Publisher; 2002. p. $67-75$.

13. Ben-Zur H, Zeidner M. Coping patterns and affective reactions under community crisis and daily routine conditions. Anxiety, Stress Coping. 1995;8:185-201.

14. Valizadeh I, Zamanzadeh V, Rahiminia E. Comparison of anticipatory grief reaction between fathers and mothers of premature infants in neonatal intensive care unit. Scand J Caring Sci. 2013;27:921-6.

15. Heinemann AB, Hellström-Westas L, Hedberg Nyqvist K. Factors affecting parents' presence with their extremely preterm infants in a neonatal intensive care room. Acta Paediatrica. 2013;102: 695-702.

16. Rocha G, Candeias L, Ramos M, Maia T, Guimaraes H, Viana V. Stress and satisfaction of mothers in neonatal intensive care. Acta Med Portuguesa. 2011;24(Suppl 2):157-66.

17. Gourounti K. Psychological stress and adjustment in pregnancy following assisted reproductive technology and spontaneous conception: a systematic review. Women Health. 2016;56:98-118.

18. Hall SL, Hynan MT, Phillips R, Lassen S, Craig JW, Goyer W, Hatfield RF, Cohen H. The neonatal intensive parenting unit: an introduction. J Perinatol. 2017;37:1259-64. 